\title{
STUDIES ON CRYSTAL SEMI-PERMEABLE MEMBRANE \\ I. ON THE FORMATION OF THE SEMI-PERMEABLE MEMBRANE OF CALCIUM CARBONATE
}

\author{
Sho MIYAMOTO AND KIYoshi TERAZAWA
}

Biochemical Department, Medical School, Tokyo Medical

and Dental University, Tokyo, Japan

(Received: September 30th, 1953)

\section{INTRODUCTION}

The study of semi-permeable membrane, one of the most important realms in biology, is seemingly limited within the examination of the natures of membrane made in a living body and no knowledge has been obtained at present for the formation mechanism.

A famous "Pfeffer's semi-permeable membrane" made of potassium ferrocyanide and copper sulfate in unglazed pottery cylinder by $W$. Pfeffer in 1877 is quite different from an artificial semi-permeable membrane at present, which is organized only by handling of extending high molecular substances, that is, collodium, cellophane, and various synthetic resins. No semi-permeable membrane made directly of low molecular substances has been so far experimentally reported. Even the Pfeffer's membrane is, therefore, apt to be thought a semi-permeable membrane of high molecular substance. In 1942, however, Miyamoto and Takikawa ${ }^{1)}$ discovered that a semi-permeable membrane was formed in an equilibrial state of the so-called Pringsheim's law, in the case of which magnesium chloride or calcium chloride was overlaid on agar with sodium silicate. Accordingly magnesium chloride solutions with various concentrations as an external electrolyte were overlaid on agar gel made in testtubes which included sodium silicate as an internal electrolyte, at the time of which the silicate layer proceeded for the inner part of lower gel with a border surface as a base between the agar gel and the solution, in the case of the external electrolyte comparatively larger in concentration than the internal, and on the contrary in the smaller case, the layer proceeds for the upper solution. In the intermediate case between them, however, there exists an equilibrium point, where there is little permeation because the precipitate forms a thin membrane on the border surface. In despite that pigment accidentally generated at the time of making the agar including sodium silicate in these experiments diffuses into the solution, upper, at both the above and under points except the equilibrial one, there is no diffusion contrarily at the equilibrial point, where the precipitate is the thinnest, found Miyamoto and Takikawa'), and further experiments were carried out as follows. The diffusing pigment in the 
above mentioned experiment comes accidentally into generation in the agar. When the precipitation proceeded adequately to overlay an upper solution on the agar, which were turned into gel immediately without coloration, and the upper solution was abandoned and replaced by Congo-red-methylene bule solution, which were overlaid, it was recognized that a semi-permeable membrane was formed contrarily without pigment permeation into gel at the equilibrium point, where it seems that there is no change on the contact surface, in despite that the pigment considerably permeated into gel at the point except the equilibrium where thick precipitate was formed. Moreover, when a precipitate of calcium silicate was formed replacing the external electrolyte with calcium chloride by the same method also, appearance of precipitating is about the same like the state in the case of the previously mentioned magnesium silicate, and the formation of semi-permeable membrane was observed at the special equilibrium point in this case. It was thus recognized that the agar pigment and haemoglobin did not permeated only at the equilibrium point, and sodium picrate and copper sulfate permeated.

This study, however, having being so far interrupted by the war and so on, has been taken up again in this department. These authors have a great deal of interest in the problem and took its charge.

Issues at this study in the first place exist in the question of whether the formation of these semi-permeable membranes is peculiar to silicate or widely explaining, all which form water-insoluble salts can generate the membrane. In order to resolve these issues, precipitate of either calcium carbonate or barium sulfate, water-insoluble was caused by using either $\mathrm{Ca}^{++}$and $\mathrm{Cl}^{-}$or $\mathrm{Ba}^{++}$and $\mathrm{SO}_{4}^{--}$. This report ascertains the fact of the formation of the so-called "crystal semi-permeable membrane" in $\mathrm{Ca}++--\mathrm{CO}_{3}^{--}$system.

\section{EXPERIMENTAL}

A series of these experiments were carried out according to the following plan: Agar (an article sold by the municipality streak-like) gel as a dispersion medium was used, into which a certain quantity of sodium carbonate $\left(\mathrm{Na}_{2} \mathrm{CO}_{2}\right)$ was dissolved in the first place as an internal electrolyte, because an aim of this experiment was taken at the study on whether a phenomenon of the socalled "semi-permeable membrane" formation is peculiar in silicate or can be generated also in the other water-insoluble salts (sodium carbonate, calcium chloride and so on). On the other hand, calcium chloride $\left(\mathrm{CaCl}_{2} \bullet \mathrm{H}_{2} \mathrm{O}\right)$ solution as an external electrolyte was used, which was changed gradually in concentration and overlaid on the agar with sodium carbonate. Examination was made thus on whether or not a semi-permeable membrane was expectedly formed on the contact surface of the electrolytes. The method was decided to observe whether Congo-red dissolved in advance in the agar gel together with the internal electrolyte can diffuse into the upper solution or on how much it is in its 
degree if diffused. Further observation was made on both each appearance of the diffusion and each state of the precipitation in the contact surface between the internal and external electrolytes. Another several series of such an experiment different in concentration in the internal solution were carried out in parallel to a series of these ones, and relations among those series were together observed. Speaking in other words, a wide combination table of the action concentrations between the internal and external electrolytes necessary for a serial experiment like this was serially arranged in this report according to the concentration of internal electrolyte as showed in Table I.

Speaking the order of experiment in detail, agar colloid solution was heated to be made with vaporization as little as possible by using a cooling tube in water bath nearing $100^{\circ} \mathrm{C}$ and quantity of sodium carbonate countered to be a certain constant in concentration as an internal solution was added to the agar. After its complete dissolution and mixture, Congo-red, an indicator of diffusion, was added. The agar thus obtained, including sodium carbonate and Congo-red, was filtered by gauze to exclude rough and impure substances intermixed, and $10 \mathrm{cc}$. of the sol was put into twenty tubes which were completely water-washed and dried up. A series are obtained by overlaying solutions of calcium chloride changed graditionally in concentration on the sodium carbonate agar gel, lower layer. In this experiment, five series were taken, $1 \%, 2 \%, 3 \%, 4 \%$, and $5 \%$ in the concentration of sodium carbonate, lower layer. The added Congo-red was fixed to $0.5 \%$, and the agar constantly $2 \%$ in these series. Adequate parts for each series of the internal electrolyte, as shown in Table I, were used in concentration, gradually changed, of calcium chloride, upper layer, related to the parts of equilibrium point in the concentration of sodium carbonate in gel. The upper threshold of the series was limited to $5 \%$, because above $5 \%$ causes a great influence on the agar.

The result was observed, after each serial tubes was scaled by rubber cork after overlaying the external electrolyte, and put untouchedly for one week in incubator fixed at $30^{\circ} \mathrm{C}$ to let the internal and external electrolytes completely proceed a reaction.

At last, the concentration of Congo-red, which diffuses into the upper solution was quantitatively measured by colorimetry of the upper solution excluding its centrifugation by the means of electrophotometer.

\section{EXPERIMENTAL RESULTS}

(I) States of Precipitation: The state of precipitation was divided into three as follows, generally observing each series:

1) In the case of thin upper solution: Within a small extent, in the concentration of upper calcium chloride solution for that of the lower solution, the precipitation proceeds for the upper solution from the overlay surface and adheres to the test tubes wall, as minute crystals. At this time, the minute 
crystals is thickly concentrated around the border surface (Fig. 1, A; Figs. 2-6, Mark: ! ).

2) In the case of thick upper solution: In a part of high concentration of the upper calcium chloride solution for the lower the precipitation proceeds for lower gel, equally with the surface as a starting point, and is formed thickly in parallel to the increase of calcium chloride concentration (Fig. 1, C, Figs. 2-6, Mark: $i)$. In the series of $1 \%, 2 \%$, and $3 \%$, however, a few of minute crystals adhere to the upper layer walls of test tube near the border surface when the concentration approaches to the equilibrium point as nextly mentioned. In the series of high concentration of sodium carbonate as $4 \%$ and $5 \%$, and in parallel, in the thick parts of calcium chloride of the upper layer, an unequal speed for the precipitation to proceeds for lower gel is observed in a few cases. Such a fact is scheduled to be stated later.

3) Equilibrium point: In the intermediate or balanced concentration between the precipitates for the upper solution and one for the lower gel, an equilibrium point was shown, where the precipitation formed a thin layer and proceeded neither for the upper nor lower (Fig. 1, B; Figs. 2-6, Mark $\rightarrow-$ ). A minute observation indicated a few of minute crystal adhering to the upper layers test tube wall near the border surface in the $1 \%$ series. Therefore, a true equilibrial state did not exist here but very near here is, it was recognized. In other words, an absolute equilibrium point called by Pringsheim seems to be within a very limited extent in $1 \%$ series, where a true equilibrium point was very difficult to be formed, because of falling always into either more or less, even with the concentration variously changed. In each series above $2 \%$, an absolute equilibrium point was observed and the larger the concentration of the series is, the wider the extent of the equilibrium point is (Figs, 2-6).

(II) Diffusion of Congo-red:

a) A phenomenon of semi-permeable membrane at the equilibrium point: In 1-4\% series as shown in Figs. 2-5, Congo-red dissolved in gel in advance permeated through the precipitate layer and colored the solution to crimson in both sides above and under the equilibrium point where the precipitation proceeded for the upper or for the lower (Photograph 1, a, c). Its colorization was similar to the control (water was used to be overlaid) in degree. In the equilibrium point where the precipitate layer is very thin or hardly recognized in the surface, the colorization degree considerably lowered than one in thick precipitate layer of the both sides (Photograph 1, b). Pigment can hardly permeate only at the equilibrium point without precipitate, while the pigment diffused and permeated where the precipitate proceeded for the upper or for the lower.

b) Difference in the state of Congo-red diffusion in both sides with the equilibrium point as a center: Examinating the state of Congo-red diffusion 
together in each series, colorization was larger nearer the surface similarly to the control (water was used) where the precipitate proceeded for the upper but the upper solution was colorized equally where it proceeded for the lower.

c) On the precipitation of Congo-red in high concentration parts of calcium chloride: In the part of very highly concentrated calcium chloride where the precipitate proceeded for the lower, the upper solutions coloration was observed to lower again contrary to the concentration of calcium chloride. The phenomenon is because thickly dispersed $\mathrm{Ca}++$ caused the precipitation of Congo-red in the precipitation layer already formed in the gel and belongs to a category different from the aim of this experiment. Fig. 6 in $5 \%$ series, indicats seemingly a quite different apperance from the former series, because the pigment was diffused where the precipitate proceeded for the lower gel. Similarly to the phenomenon in 1-4\% series, $\mathrm{Ca}^{++}$is presumed to have caused the precipitation of Congo-red in the precipitate layer.

(III) Appearance of a pseudo-equilibrium point except the ture equilibrium point in highly concentrated series, and "chemical garden": In $4 \%$ and $5 \%$ series, a phenomenon, which is unable to be observed in the under concentrated series, appears; In the part in which the precipitate is to proceed for the lower gel, facts were observed that the precipitation without an equal proceeding, was interupted in its process, to be an irregular concavity in proceeding surface, formed a thin membrane seemingly like in the equilibrium point, for which it did not proceed for either side, and the former two phenomena were together caused (Fig. 7). There is no definite knowledge on the mechanism at present, which comes causes these facts. A detailed observation, however, indicated that there is a thin mantle membrane on these test tubes under the precipitate layer, by which the reaction is seemed to be have been interupted all at once. At a sight, another equilibrium point is apt to be thought to have appeared in these parts. No definite explanation has been obtained on the mechanism about why such an equilibrial phenomenon appeared at the point except the true Pringsheim's equilibrium point only when highly concentrated electrolytes, both internal and external, coexisted and in the part where the precipitation once began to proceed for the lower gel. These investigators suppose the following case as a possible mechanism as a result of an observation of these phenomena; As often stated, a phenomenon of this kind never occurs in the case of lowly concentrated lower solution $\left(\mathrm{Na}_{2} \mathrm{CO}_{3}, 1-3 \%\right)$, but does only in the case of higher-concentrated lower solution than a certain limit (above $4 \% \mathrm{Na}_{2} \mathrm{CO}_{3}$ ). It is, therefore, first an essential condition for the appearance of these phenomena that highly concentrated solutions, both internal and external, come into contact. When both thick solutions come into contact, a severe reaction in the contact surface causes a great deal of the precipitate on the surface and its under part. When the precipitate is made 
under such a condition, a large crystal supposedly is accidentally formed by some chances. In this case of course, because calcium chloride above the crystal is thicker in concentration than lower layer's sodium carbonate, the former will begin to diffuse under through crack among the crystals. Under such a condition quite different from the formerly mentioned case, the diffusion at this time proceeds hemispherically with the erack as a center. On the other hand, the concentration of sodium carbonate under the crystal suddenly decreases around it temporarily, similarly as seen in the case of the Liesegang's phenomenon, when such a large one is once formed. Following diffusion of sodium carbonate into the upper part from under to supply its sudden decrease comes into contact again with that of calcium chloride from upper. It is easily to be supposed at this time that a mantle membrane may be balanced in the socalled "Pringsheim's equilibrium" to be formed.

The above-mentioned explanation is made quite on a hypothesis as already stated, and the dissolution of these issues is dependent upon the experiment in future. Anyhow, interest is absorbed in the fact that there appear the 2nd and 3rd equilibrium states except the true Pringsheim's equilibrium in highly concentrated solutions, both internal and external.

It is often observed, moreover, that there appear one to several bacillary processes from the surface of membrane contact with the test tube wall in still larger concentration of calcium chloride. The most of these processes grow little by little and at last reaches near the surface of upper solution. This phenomenon is thought to resemble to the so-called "chemical garden" by Samejima (Fig. 7). In $4 \%$ series a few of these appeared but in 5\% many did.

\section{Discussion AND SUMMARY}

As stated in the introduction, in order to investigate whether the fact that the nature of semi-permeable membrane is shown by this membrane of silicate at the Pringsheim's equilibrium point, with which Miyamoto and Takikawa accidentally met, is a phenomenon peculiar in silicate or common in the other supstances forming water-insolble salts also, the experiment of calcium carbonate was in the first place carried out. It was recognized that such a phenomenon can be similarly generated. There have been no definite knowledge on the state of crystal in the border surface at this time yet, but a probable gathering of minute crystals is thought to form a semi-permeable membrane. Miyamoto and $\mathrm{Okuda}^{3}$ ) observed ever that sodium chloride crystal in gelatine is quite influenced at the rate of the concentration of gelatine for table salts and by the thickness of the layers also. Of all, in the case of both thin solutions of gelatine and table salts, the salts is crystalized in a thin layer after dried up, in the case of which the salts crystal, generated to be originally scattered in a regular hexahedron, was pressed and crashed to become just feather-like and to form a membrane made of the crystal with the close feather, observed 
they. There is no proof on whether or not the crystal of the border surface in the experiment if formed by the above-mentioned way, but judging from the former experiment by Miyamoto and Okuda, in the case of crystallization in such a very thin layer, the crystal seems to be pressed and crashed like a mica as mentioned above and a membrane seems to be formed with each scrap closely in contact, it is thought. In this viewpoint, these authors want to call this phenomenon "crystal semi-permeable membrane".

It is of a great interest that such a crystal forms such a membrane. Judging from the experiment by Miyamoto and Okuda, however, it seems essential for the phenomenon of the crystal changing into a membrane that it is formed in a very thin layer. If it is thick, crystal will be despersed separately and incontinuously but if thin, because a regular crystal was pressed and crashed to be extended at last, each crystal comes into contact. It seems to be one of the most important conditions for the membrane formation by the crystal that it is formed in a thin layer. While in the case of the experiment by Miyamoto and Okuda, crystal was pressed and crashed mechanically in the case of the experiment by Miyamoto and Takikawa and in their study, these thin membranes of crystal are thought to be formed on the border surface by a balanced state of osmotic pressure at the Pringsheim's equilibrium point.

In the other hand, no proof has been, at present, obtained to explain the above-mentioned thinking way based on a hypothesis. In comparison of the appearance of crystal in the contact surface at the so-called presumed equilibrium point to that very apart from the equilibrium point, there were some phenomena useful for supporting the hypothesis. From the concentration series of the upper solution the test-tube was taken up, in which semi-permeable membrane is thought to be formed between above and under layers at the so-called presumed equilibrium point, and after the upper solution was run away quietly, the surface of agar of the lower layer, contact surface between above and under layers, was thinly cut. A microscopic photograph was taken by reflective light for the appearances of crystal. In contrast to this, the surface of agar at a point very apart from the equilibrium point was similarly photographed and compared (Photograph 2). Observing these pictures, both in the part of the presumed equilibrium point and in that very apart from the point, considerably large crystals were scattered. In the parts of the presumed equilibrium point, there appears a dispersion light like hallation entirely between scattered large crystal. On the contrary, in the parts apart from the equilibrium point, each large erystal is separated with the other and its gap is deeply transparent.

Much attention must be paid to the fact that the equilibrium point attained may be near the real equilibrium point within the series of experience, but not a real one as a result of an experience by the authors. That is to say that the real equilibrium point exists on a conception because in the experi- 
ment, the concentration series are developed on the basis of dilution at the equal intervals, but it is impossible for the point to be brought on a test tube.

Taking those into consideration and examining those two sheets of photograph, it is thought that perhaps very small crystals will be arranged at the equilibrium point in a very thin layer and very closely, may be touched each other. But such a thought unless the form and structure of crystals are cleared, is nothing but a guess.

In this study, the authors want to confirm the phenomena to be brought by experience with use of other sorts of salts, that is, 2-2 salt $\left(\mathrm{BaSO}_{4}\right)$ and to go to clearing of the arrangement and structure of small crystals at the point.

\section{CONCLUSION}

1) When internal elctrolyte and external one make water-insoluble salts, at the point of balanced concentration, Pringsheim's equilibrium point, those water-insoluble salts made a semi-permeable membrane not only in the case of silicate Miyamoto and Takikawa previously reported but similarly in calcium carbonate.

2) So, under the proper conditions, generally speaking, water-insoluble salts are seemingly considered to make a semi-permeable membrane. Those membranes, at least those membranes which were made in the case of calcium silicate and calcium carbonate, were decided to be called "crystal semi-permeable membrane" by Miyamoto and Terazawa.

\section{BIBLIOGRAPHY}

1) Miyamoto, S., and Takikawa, ., Igaku to Seibutsu Gaku (Medicine and Biology, in Japanese), p. 351 (1942).

2) Freundlich, H., Kapillarchemie, Band II. s. 714.

3) Miyamoto, S., and Okuda, ., Igaku to Seibutsu Gaku (Medicine and Biology, in Japanese), p. 6 (1944). 
Table 1

\begin{tabular}{|c|c|c|c|c|c|c|c|c|c|c|}
\hline 㧜 & & I & & II & & III & & IV & & V \\
\hline 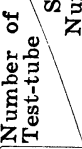 & \begin{tabular}{|l} 
Inter- \\
nal elec- \\
trolyte \\
(gel \\
layer)
\end{tabular} & \begin{tabular}{|c} 
Exter- \\
nal elec- \\
trolyte \\
(over \\
lay)
\end{tabular} & \begin{tabular}{|c|} 
Inter- \\
nal elec- \\
trolyte \\
(gel \\
layer)
\end{tabular} & \begin{tabular}{|c} 
Exter- \\
nal elec- \\
trolyte \\
(over \\
lay)
\end{tabular} & \begin{tabular}{|c} 
Inter- \\
nal elec- \\
trolyte \\
(gel \\
layer)
\end{tabular} & $\begin{array}{c}\text { Exter- } \\
\text { nal elec- } \\
\text { trolyte } \\
\text { (over } \\
\text { lay) }\end{array}$ & \begin{tabular}{|l} 
Inter- \\
nal elec- \\
trolyte \\
(gel \\
layer)
\end{tabular} & $\begin{array}{c}\text { Exter- } \\
\text { nal elec- } \\
\text { trolyte } \\
\text { (over } \\
\text { lay) }\end{array}$ & $\begin{array}{c}\text { Inter- } \\
\text { nal elec- } \\
\text { trolyte } \\
\text { (gel } \\
\text { layer) }\end{array}$ & $\begin{array}{c}\text { Exter- } \\
\text { nal elec- } \\
\text { trolyte } \\
\text { (over } \\
\text { lay) }\end{array}$ \\
\hline 1 & 1 & 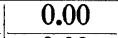 & 2 & 0.00 & 3 & $\begin{array}{l}0.00 \\
\end{array}$ & 4 & 0.00 & 5 & $\begin{array}{ll}0.00 \\
\end{array}$ \\
\hline 2 & $"$ & 0.02 & " & 0.04 & " & 0.04 & " & 0.04 & 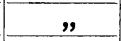 & 0.05 \\
\hline 3 & " & 0.12 & 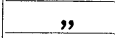 & 0.24 & $\#$ & 0.24 & 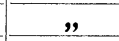 & 0.24 & " & 0.30 \\
\hline 4 & $"$ & 0.22 & " & 0.44 & " & 0.44 & " & 0.44 & $"$ & 0.55 \\
\hline 5 & " & 0.32 & " & 0.64 & $"$ & 0.64 & " & 0.64 & 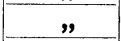 & 0.80 \\
\hline 6 & 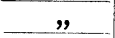 & 0.42 & " & 0.84 & 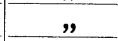 & 0.84 & $"$ & 0.84 & $"$ & 1.05 \\
\hline 7 & 川 & 0.52 & " & 1.04 & $"$ & 1.04 & 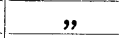 & 1.04 & 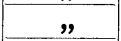 & 1.30 \\
\hline 8 & 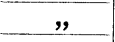 & 0.62 & 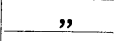 & 1.24 & ” & 1.24 & $"$ & 1.24 & " & 1.55 \\
\hline 9 & $"$ & 0.72 & $"$ & 1.44 & " & 1.44 & " & 1.44 & " & 1.80 \\
\hline 10 & " & 0.82 & $\#$ & 1.64 & $"$ & 1.64 & $\#$ & 1.64 & 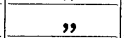 & 2.05 \\
\hline 11 & " & 0.92 & $"$ & 1.84 & " & 1.84 & " & 1.84 & " & 2.30 \\
\hline 12 & " & 1.02 & 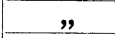 & 2.04 & \# & 2.04 & 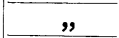 & 2.04 & $"$ & 2.55 \\
\hline 13 & " & 1.12 & , & 2.24 & 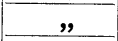 & 2.24 & " & 2.24 & " & 2.80 \\
\hline 14 & " & 1.22 & , & 2.44 & 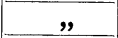 & 2.44 & $"$ & 2.44 & " & 3.05 \\
\hline 15 & ״ & 1.32 & " & 2.64 & " & 2.64 & $"$ & 2.64 & $\#$ & 3.30 \\
\hline 16 & 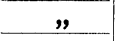 & 1.42 & 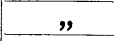 & 2.84 & " & 2.84 & 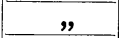 & 2.84 & $"$ & 3.55 \\
\hline 17 & " & 1.52 & & & " & 3.04 & " & 3.04 & " & 3.80 \\
\hline 18 & $"$ & 1.62 & & & \# & 3.24 & 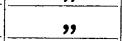 & 3.24 & $"$ & 4.05 \\
\hline 19 & " & 1.72 & & & $"$ & 3.44 & \# & 3.44 & $"$ & 4.30 \\
\hline 20 & " & 1.82 & & & " & 3.64 & " & 3.64 & " & 4.55 \\
\hline 21 & $\#$ & 1.92 & & & $\#$ & 3.84 & $"$ & 3.84 & $\#$ & 4.80 \\
\hline 22 & & & & & & & 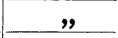 & 4.04 & ", & 5.05 \\
\hline 23 & & & & & & & " & 4.24 & " & 5.30 \\
\hline 24 & & & & & & & $"$ & 4.44 & " & 5.55 \\
\hline 25 & & & & & & & $"$ & 4.64 & 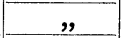 & 5.80 \\
\hline 26 & & & & & & & & 4.84 & & \\
\hline
\end{tabular}
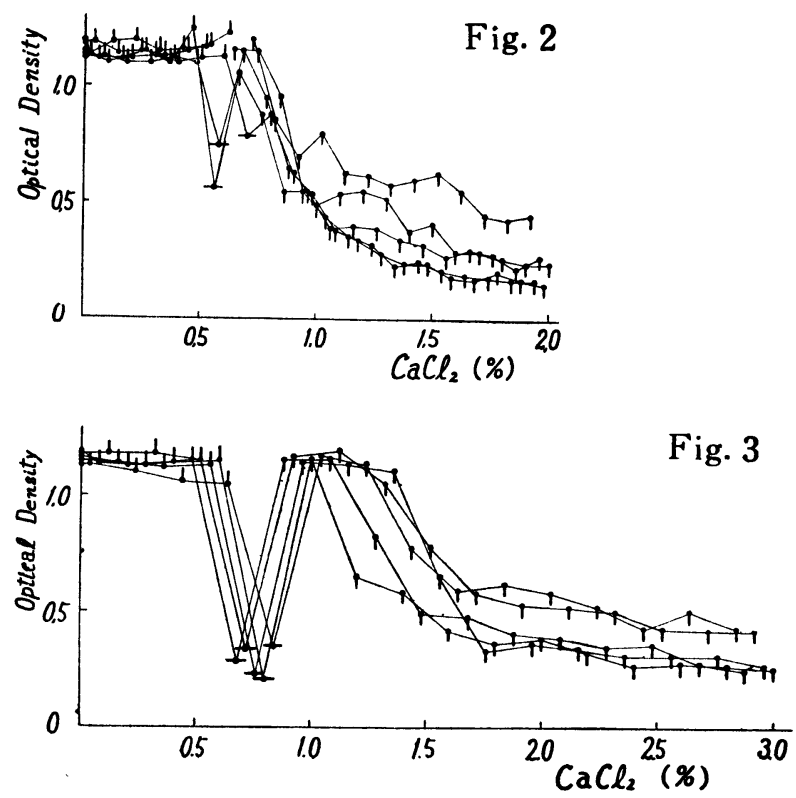

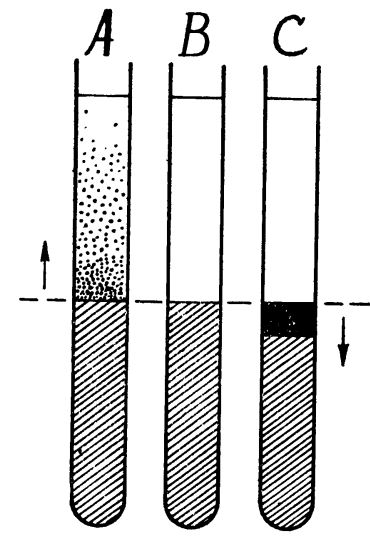

Fig. 1 

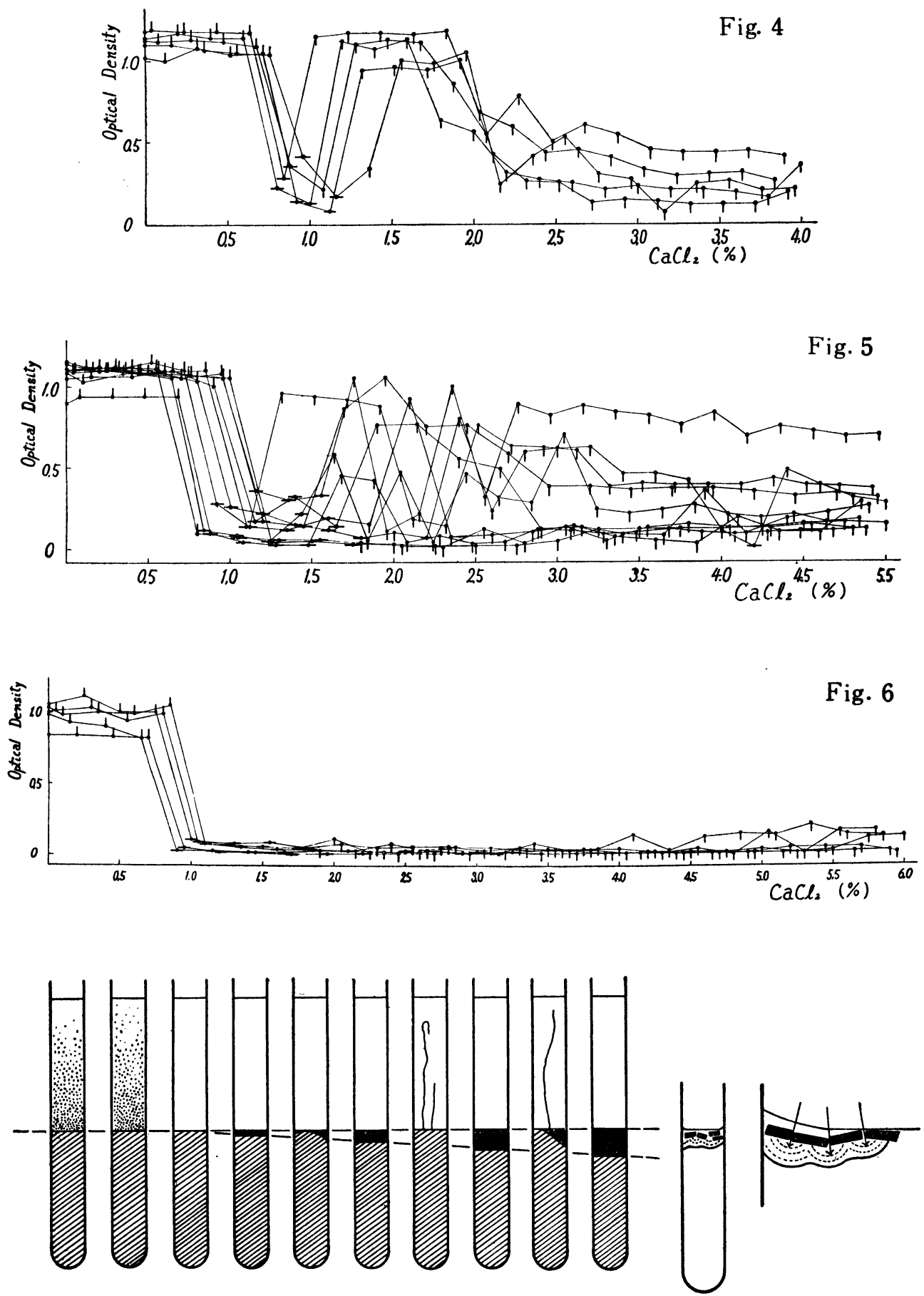

Fig. 7

Fig. 8 


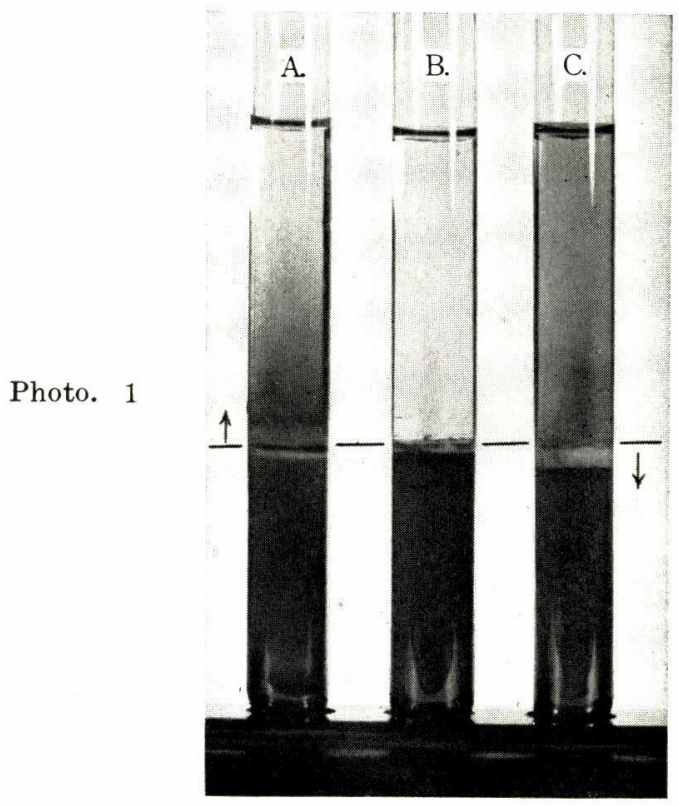

Photo. 2
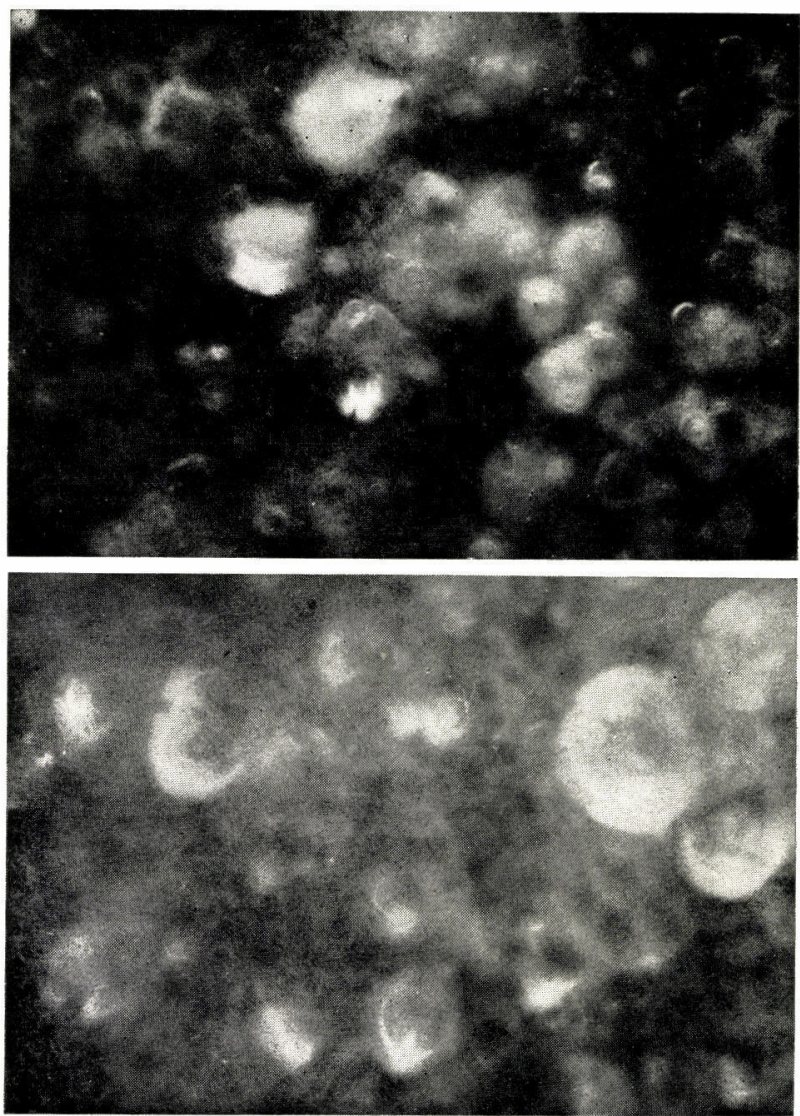